\title{
Intra-arterial Balloon Occlusion to Reduce Operative Bleeding for Placenta Previa Accreta Spectrum
}

\author{
Yoshihiko Murayama, MD, $\mathrm{PhD}^{1}$ Hiroyuki Seki, MD, $\mathrm{PhD}^{2}$ Satoru Takeda, $\mathrm{MD}, \mathrm{PhD}^{3,4}$
}

1 Department of Obstetrics and Gynecology, Nerima Hikarigaoka Hospital, Tokyo, Japan

2 Department of Obstetrics and Gynecology, Saitama Medical Centre, Saitama Medical University, Kawagoe, Japan

3 Department of Obstetrics and Gynecology, Faculty of Medicine, Juntendo University, Tokyo, Japan

${ }^{4}$ Aiiku Research Institute for Maternal, Child Health, and Welfare, Imperial Gift Foundation Boshi-Aiiku-Kai, Tokyo, Japan

\begin{abstract}
Address for correspondence Yoshihiko Murayama, MD, PhD, Department of Obstetrics and Gynecology, Nerima Hikarigaoka Hospital, 2-11-1, Hikarigaoka, Nerima-ku, Tokyo, Japan 179-0072 (e-mail: ymhiko@saitama-med.ac.jp).
\end{abstract}

Surg J (NY) 2021;7(suppl S1):S11-S19.

\begin{abstract}
Keywords

- cesarean hysterectomy

- common iliac artery balloon occlusion

- intra-arterial balloon occlusion

- internal iliac artery ligation

- placenta previa accreta spectrum

Cesarean section for placenta previa accreta spectrum carries a significant risk of massive hemorrhage. Hence, it is necessary to understand the various hemostatic procedures, damage control surgery and resuscitation for massive hemorrhage, and systemic management against hypovolemic shock and coagulopathy. In cases of placenta previa with previous cesarean section, the operation should be performed in a tertiary medical facility with well-trained staff and blood availability for transfusion. Preoperative placement of an intra-arterial balloon occlusion catheter in the common iliac artery or aorta is useful for preventing massive hemorrhage.
\end{abstract}

\section{Surgical Steps}

1. Insert the catheter for epidural anesthesia.

$\downarrow$

2. Insert the sheath introducer from both sides of the inguinal region and place the balloon in the common iliac artery.

$\downarrow$

3. Place the patient in the surgical position and check vital signs and monitors.

$$
\downarrow
$$

published online September 17, 2021
DOI https://doi.org/ 10.1055/s-0040-1721491. ISSN 2378-5128.
4. Insert the catheter in both ureters to prevent ureteral damage during the surgery. $\downarrow$

5. Grasp the uterine cervix with the forceps transvaginally. $\downarrow$

6. Induce general anesthesia. $\downarrow$

7. Start the cesarean section with a vertical abdominal incision. $\downarrow$

8. Before incising the uterus, confirm the placental margin by ultrasonography to determine the incision site. $\downarrow$ (c) 2021. The Author(s).

This is an open access article published by Thieme under the terms of the Creative Commons Attribution-NonDerivative-NonCommercial-License, permitting copying and reproduction so long as the original work is given appropriate credit. Contents may not be used for commercial purposes, or adapted, remixed, transformed or built upon. (https://creativecommons.org/ licenses/by-nc-nd/4.0/)

Thieme Medical Publishers, Inc., 333 Seventh Avenue, 18th Floor, New York, NY 10001, USA 


\section{Make a uterine incision and deliver the baby.}

$\downarrow$

10. Decide whether a consecutive hysterectomy is necessary.

$\downarrow$

11. Cut and ligate the round ligament and then the proper ligament of the ovary and the fallopian tube. Expand the retroperitoneal space.

$\downarrow$

12. Cut and ligate the uterine artery and vein, and then cut and suture the uterosacral ligament and the cardinal ligament. $\downarrow$

13. Infuse 3,000 units of unfractionated heparin intravenously, immediately before balloon dilation. $\downarrow$

14. Dilate the balloon in both common iliac arteries. $\downarrow$

15. Confirm that the internal iliac artery pulsation becomes weak in the surgical field and that the pulse wave attenuates but does not disappear completely using oxygen saturation monitors attached to both the big toes. $\downarrow$

16. Separate the urinary bladder from the uterus. $\downarrow$

17. Perform hysterectomy and close the amputation stump of the vagina. $\downarrow$

18. Deflate the balloon.

$$
\downarrow
$$

19. If necessary, use protamine sulfate for heparin neutralization.

$\downarrow$

20. Search for bleeding points and stop them. Close the incision of the abdomen.

$\downarrow$

21. Remove the balloon and sheath introducers.

\section{Cesarean Section Strategy for Placenta Previa Accreta Spectrum with Intra-arterial Balloon Occlusion}

In the cases of placenta previa accreta spectrum (placenta previa complicated with placenta accreta, increta, or percreta), obstetricians may be required to make the difficult decision of performing a hysterectomy during the cesarean section. ${ }^{1-3}$ However, cesarean hysterectomy $(\mathrm{CH})$ in these cases may occasionally be accompanied by fatal massive bleeding.

Our hospital has had the experience of managing 60 patients with placenta previa accreta who had undergone $\mathrm{CH}$ with diagnosed invasion of the placenta pathologically. After examining these cases retrospectively, we found that we had encountered cases of massive bleeding in association with placenta percreta and placenta accreta when we tried to separate the placenta from the uterus. Furthermore, we attempted to ligate the internal iliac artery in some cases after delivering the baby and before starting the hysterectomy to reduce blood loss. However, we could not find any statistically significant differences in blood loss between the cases with or without the ligation. In unsuccessful cases, it was observed that collateral blood flow to the uterus had immediately developed after the internal iliac artery ligation. ${ }^{4}$ In addition, it has been reported that using internal iliac artery balloon occlusion with $\mathrm{CH}$ could control bleeding rapidly compared with ligation; however, prophylactic intravascular balloon catheters did not benefit women with placenta accreta undergoing $\mathrm{CH}^{5}$ These results suggest that we may not be able to control the hemorrhage volume of $\mathrm{CH}$ by blocking the internal iliac artery because of collateral blood flows.

In contrast, there is another strategy in which after delivering the baby, the obstetricians close the surgical incision, including the uterus, without separating the placenta and then perform hysterectomy several weeks later. ${ }^{6}$ This is a very sensible method because we can start hysterectomy after the blood flow of the uterus has decreased. However, if the placenta separates unexpectedly during the first surgery, we might not be able to avoid the risk of massive bleeding.

Shih et al published a case report describing that they performed $\mathrm{CH}$ using common iliac artery balloon occlusion (CIABO) to reduce the hemorrhage volume in a patient with placenta percreta. ${ }^{7}$ Since then, we have started using CIABO for $\mathrm{CH}$ and have had 30 cases since 2005. We succeeded in significantly decreasing the hemorrhage volume in these cases using $\mathrm{CIABO}$ for $\mathrm{CH}$ rather than internal iliac artery ligation. ${ }^{8,9}$

Recently, advances in medical device engineering have made the balloon and sheath introducer thinner ( $\leq 10 \mathrm{Fr}$ ), which has allowed for safer balloon placement inside the abdominal aorta. Therefore, this has contributed to an increased number of reports on the usefulness of abdominal aorta balloon occlusion ( $A A B O)$ to reduce blood loss in patients undergoing $\mathrm{CH} .{ }^{10} \mathrm{AABO}$ has the advantage that it needs only one sheath introducer for the occlusion balloon catheter; however, it also has the disadvantage that the balloon cannot be expanded before $\mathrm{CH}$, and it may block the branch of the inferior mesenteric artery (-Fig. 1).

\section{Preoperative Preparation}

The patients in whom placenta previa accreta is strongly suspected would be managed during hospitalization after 30 weeks of gestation. Four hundred milliliter of blood is generally collected under the observation of fetal heart rate patterns and uterine contractions from 3 weeks prior to the planned date of cesarean section, and a total of $1,200 \mathrm{~mL}$ of blood is stored for autologous blood transfusion. Owing to the strong collaboration with other specialists, including pediatricians, radiologists, urologists, and anesthesiologists, we usually plan to perform $\mathrm{CH}$ at approximately 35 to 36 weeks of gestation. If the circumstances of each hospital do not allow this schedule, $\mathrm{CH}$ may be planned at 34 weeks of gestation because we should avoid performing $\mathrm{CH}$ in an emergency situation as much as possible. 


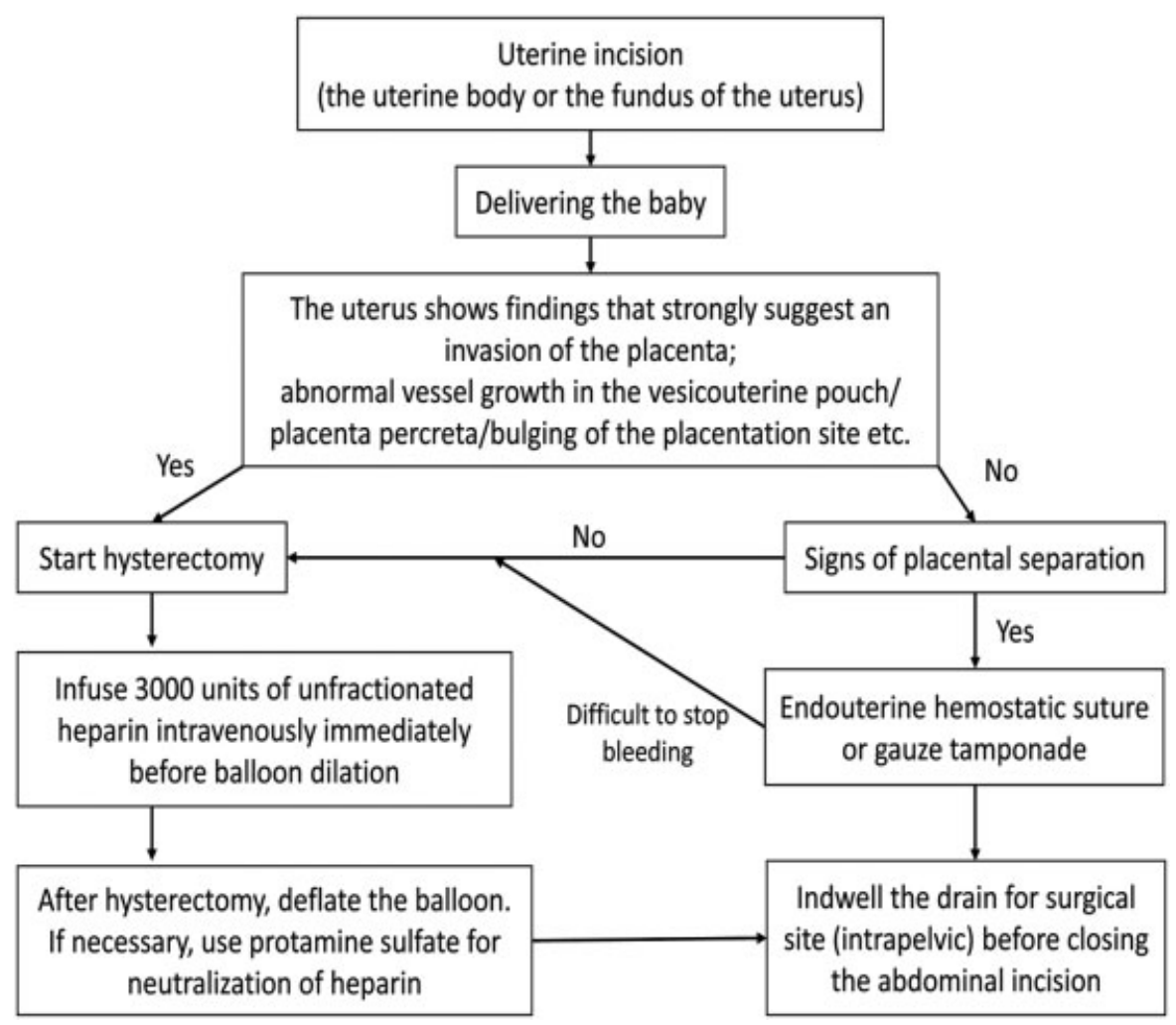

Fig. 1 Cesarean section strategy for placenta previa accreta spectrum using common iliac artery balloon occlusion.

\section{Informed Consent}

We need to explain the following details to the patients and their families and inform them our outcomes using $\mathrm{CH}$ with CIABO; written informed consent is then obtained before hospitalization. Among the cases in which the placenta is on a prior uterine scar, half of the cases develop complicated placenta accreta pathologically. Accurate preoperative prediction of placenta accreta is said to be difficult. Therefore, we often decide whether the hysterectomy is necessary, depending on the actual perioperative findings inside the abdomen. In some cases of hysterectomy, placenta accreta may not be diagnosed by pathology. Depending on the findings during the surgery, we may decide to end it with the placenta still inside the uterus and perform an arterial embolization using the already-available catheter. In this case, we will plan to perform hysterectomy at a later date.

The patients should be informed about the details of the risks associated with inserting $\mathrm{CIABO}$, such as maternal lower limb ischemia and crush syndrome, thrombosis, inguinal hematoma, and damage to the artery.

\section{Tips and Warnings}

If a patient is eagerly hoping to avoid hysterectomy, we should understand the following:

- Separation of the placenta may cause massive bleeding. If we cannot control the bleeding by banding or pressure hemostasis, we will have to perform hysterectomy.

- It is reported that with conservative management, hysterectomy can be avoided. In this method, after the delivery of the baby, the surgery is ended with the placenta still inside the uterus, and we will wait for the spontaneous disappearance of the placenta. We have used this method for three cases; however, we ultimately could not avoid the hysterectomy because of postoperative bleeding or infection. In one of the cases, there was a postoperative massive blood loss of $17,000 \mathrm{~mL}$.

\section{Explanation of the Procedures}

1. Insert the catheter for epidural anesthesia.

Inserting the catheter for epidural anesthesia will help in pain control during the procedures of inserting the sheath introducer and balloon catheter and inserting the catheter in both ureters. However, in one of our cases, when a patient was in the lateral decubitus position, sudden heavy bleeding occurred from the uterus, requiring prompt cesarean section. From this experience, we have learned to pay attention even during the induction of anesthesia.

2. Insert the sheath introducer from both sides of the inguinal region and place the balloon in the common iliac artery. 


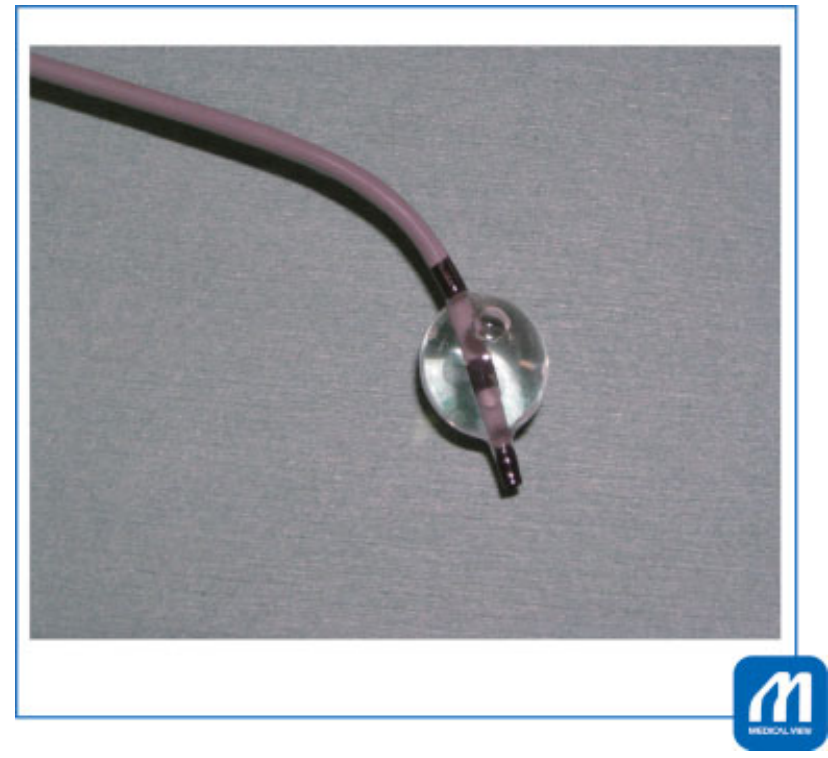

Fig. 2 Intra-arterial balloon occlusion catheter made of polyurethane resin. (Reproduced with permission from Murayama Y, Seki H, Itakura A, Takeda S. Placenta previa accreta spectrum; approach to reduce blood loss: intra-arterial balloon occlusion. In: Konishi I, Hiramatsu Y, Sakuragi N, Takeda S, eds. Surgery for Pregnancy with Placenta Previa and Placenta Accreta: Careful Preparation and Critical Management. OGS NOW, No.9 (in Japanese). Tokyo: Medical View; 2012:100-109. Copyright $\odot$ Medical View).

It is common to perform magnetic resonance imaging before the surgery in the cases of placenta previa accreta. We recommend evaluating the diameter and length of the common iliac artery. Normally, a $10-\mathrm{mm}$ diameter balloon should be adequate to occlude the artery.

When we used a balloon made of latex, we noticed that it was damaged after three placements. Therefore, we have started using a new balloon made of a polyurethane resin, which is more preferable (-Fig. 2 ).

We recommend the procedure during which the balloons are placed by crossing over to the common iliac artery on the opposite sides, that is, a catheter inserted from the right femoral artery is used to place a balloon in the left common iliac artery and a catheter inserted from the left femoral artery is used to place a balloon in the right common iliac artery (-Fig. 3). This is done because after delivering the baby, the position of the balloons may have changed to a more peripheral side than expected, and occasionally, the balloon may enter the external iliac artery, especially in patients who have shorter common iliac arteries. Since these balloons are inserted retrogradely, their position is easily changed because of the blood flow. Thus, this is a sensible method because the balloons are in the antegrade blood flow.

Since the baby is inside the uterus, trial dilation of the balloon should be performed one by one before surgery, and the fetal heart rate must be monitored.

$6 p t ?>$ The maximum radiation exposure was approximately $70 \mathrm{mGy}$ during this procedure. Since the exposure value of the

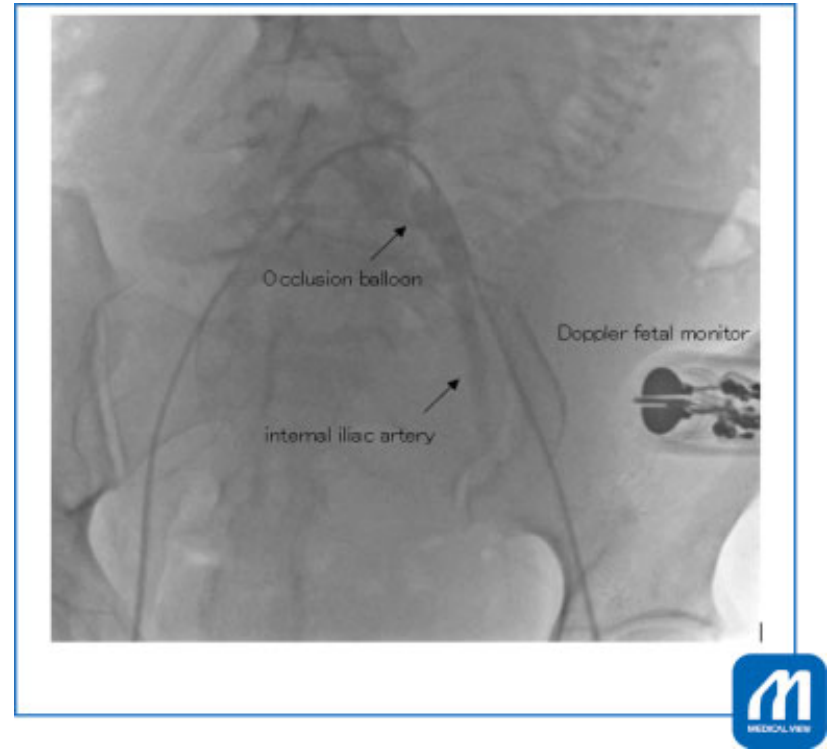

Fig. 3 The balloon in the left common iliac artery, which was inserted from the right femoral artery, is inflated. The balloon catheter placement is performed on the contralateral side to the puncture site, passing across the aortic bifurcation and placing antegradely. When inflating the balloon, do not fully inflate the balloon; instead, inflate with slight blood flow remaining toward the femoral artery to avoid excessive ischemia. In addition, it is recommended to determine the catheter placement site at slightly proximal to the target place because the balloon may move slightly toward the distal side due to blood flow. (Reproduced with permission from Murayama Y, Seki H, Itakura A, Takeda S. Placenta previa accreta spectrum; approach to reduce blood loss: intra-arterial balloon occlusion. In: Konishi I, Hiramatsu Y, Sakuragi N, Takeda S, eds. Surgery for Pregnancy with Placenta Previa and Placenta Accreta: Careful Preparation and Critical Management. OGS NOW, No.9 (in Japanese). Tokyo: Medical View; 2012:100-109. Copyright @ Medical View).

fetus is expected to be lower than this value, the balloon placement procedure can be performed under the threshold value.

In recent years, several hospitals have added a hybrid operating room that combines an operating room with a cardiocerebral vascular radiography room. If the apparatus is present in this room, the procedure might be performed after delivering the baby. However, since we have experienced three cases of massive bleeding before delivery, we think it would be better to perform this procedure before starting the surgery.

3. Place the patient in the surgical position and check vital signs and monitors.

Since there are procedures to insert the urethral catheter and mark the uterine cervix afterwards, the patients need to be placed in the lithotomy position. However, the flexion of the hip joint may cause damage to the sheath introducer and the balloon. Therefore, we place the patients in a position with legs apart and hips extended using a levitator (MIZUHO Corporation, Japan). We monitor the pulse wave and oxygen saturation of both the big toes during surgery to evaluate the effect of balloon occlusion and avoid lower-limb ischemia. Furthermore, we establish intravenous lines in both arms 
using 18- to 14-gauge needles and an intra-arterial line prepared for massive bleeding.

We prepare the autologous blood transfusion from 3 weeks prior to the planned date of cesarean section, and a total of $1,200 \mathrm{~mL}$ is stored. In addition to this, we prepare 20 units each of red blood cell concentrates and fresh frozen plasma for $\mathrm{CH}$.

4. Insert the catheter in both the ureters to prevent ureteral damage during the surgery.

The uterus of the placenta previa accreta has a swollen bottom-like structure resembling the shape of a snowman as the same as the huge cervical myoma that leaves no intrapelvic space and prevents palpation and identification of the ureters. So, you will feel that it is difficult to visually confirm the uterine arteries and the ureters. The catheters inside both the ureters make it easier to confirm the position of the ureters even in a tense situation when the bleeding increases.

In the prototype of the $\mathrm{CH}$ strategy, we performed this procedure the day before surgery. However, because of pain control and bleeding from the bladder, we have recently started performing it immediately before the $\mathrm{CH}$.

5. Grasp the uterine cervix with the forceps transvaginally.

The pregnant uterine cervix becomes softer than the nonpregnant uterus, making it difficult to palpate the position of the vaginal fornix. If the bladder was over-separated from the vagina, it would be hard for hemostasis to occur. Therefore, grasping the uterine cervix transvaginally with the grasping forceps helps to palpate the position of the vaginal fornix. We have never experienced uterine bleeding using this procedure.

6. Induce general anesthesia.

In the past, for a brief period, we tried performing $\mathrm{CH}$ by spinal anesthesia because we wanted the patients to hear the first cry of their babies. However, because of the sheath introducer and the balloons, the patients could not maintain the bent posture required for spinal anesthesia. Furthermore, we also observed that a patient suddenly started bleeding heavily from the uterus during the spinal anesthesia, requiring us to start the cesarean section urgently without using the ureteral catheters. It is important to perform the procedures exactly as planned to reduce the risks of $\mathrm{CH}$. Therefore, we perform $\mathrm{CH}$ using general anesthesia. In addition, since patients with placenta previa accreta are usually in extreme tension, general anesthesia may be considered the best choice for them.

7. Start the cesarean section with a vertical abdominal incision.

It should be a consensus that the vertical abdominal incision is a sensible method of creating enough space for $\mathrm{CH}$. Since we make the transverse incision of the fundus of the uterus (Kotsuji method) with reference to the report, ${ }^{11}$ the abdominal incision may be extended to the navel.

8. Before incising the uterus, confirm the placental margin by ultrasonography to determine the incision site.

Massive bleeding tends to occur if the placenta is detached before or soon after delivering the baby in the case of placenta previa accreta. The uterine incision should be

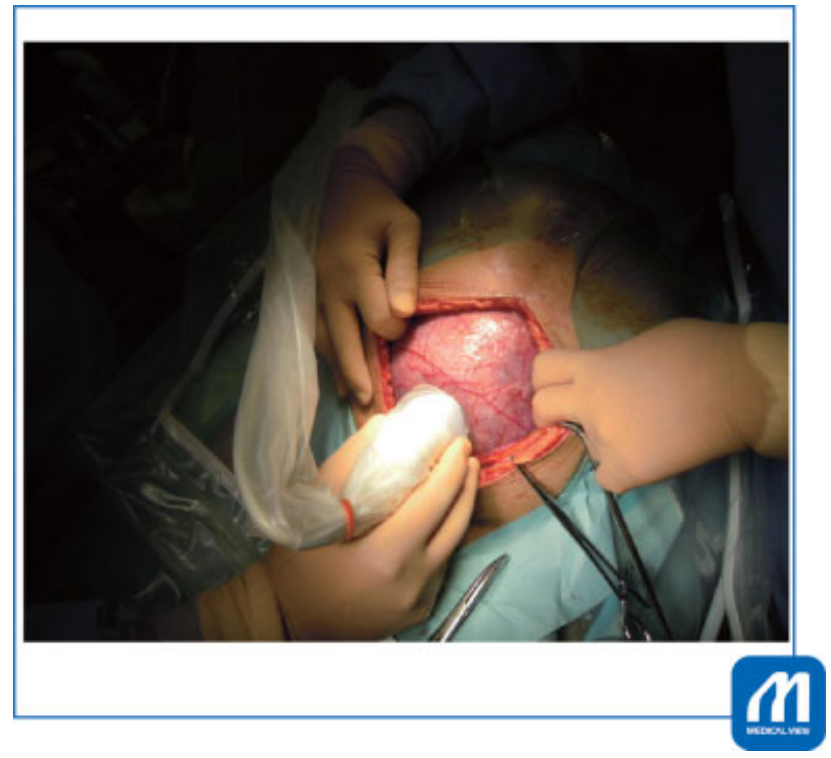

Fig. 4 Ultrasonographic image taken during cesarean hysterectomy. (Reproduced with permission from Murayama Y, Seki H, Itakura A, Takeda S. Placenta previa accreta spectrum; approach to reduce blood loss: intra-arterial balloon occlusion. In: Konishi I, Hiramatsu Y, Sakuragi N, Takeda S, eds. Surgery for Pregnancy with Placenta Previa and Placenta Accreta: Careful Preparation and Critical Management. OGS NOW, No.9 (in Japanese). Tokyo: Medical View; 2012:100-109. Copyright $\odot$ Medical View).

made superior to the placenta at some distance to avoid causing spontaneous placental detachment. After the abdominal incision, we think it is important to identify the placental margin by ultrasonography before choosing the incision site (-Fig. 4).

9. Make a uterine incision and deliver the baby.

Care should be taken if a vertical uterine incision was made because the incision may be extended to the placentation site when the baby is delivered, causing massive bleeding (-Fig. 5).

10. Decide whether a consecutive hysterectomy is necessary.

We assess the placental invasion more than the placenta increta and decide to proceed with hysterectomy when the uterus showed findings such as extensive abnormal growth of new blood vessels in the vesicouterine pouch, bulging of the placentation site with the proliferation of blood vessels, or if the placenta can be seen through the serosa.

The uterus of the strictly defined placenta accreta may not express these findings prominently. In those cases, we consider it appropriate to determine whether hysterectomy should be performed based on the presence or absence of signs of spontaneous detachment of the placenta. If the placental invasion is partial, perform the following steps before hysterectomy. First, the placenta is removed manually, while conducting pressure hemostasis with a towel or gauze covering the intrauterine surface where the placenta detached and then suturing directly at the bleeding points and/or conducting uterine compression sutures. 


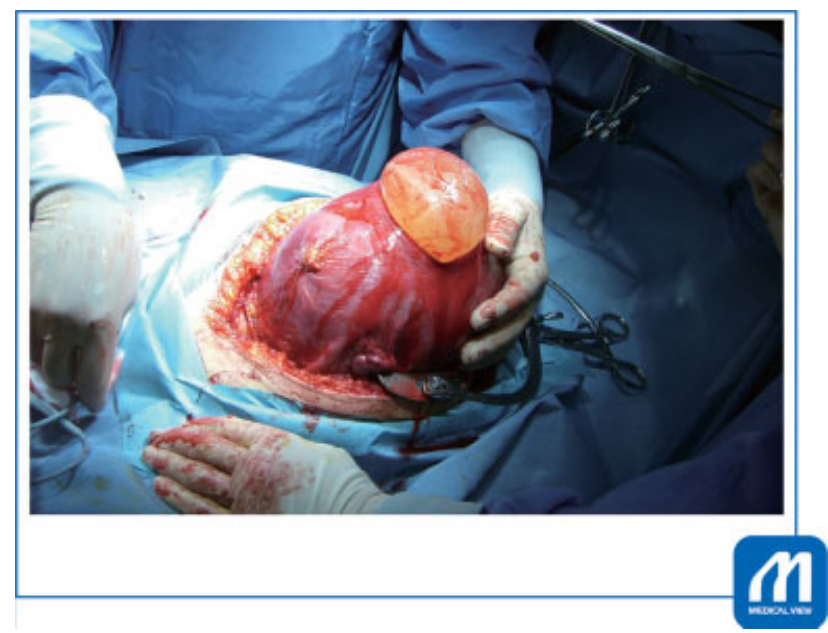

Fig. 5 Transverse incision of the fundus of the uterus (Kotsuji method ${ }^{11}$ ). (Reproduced with permission from Murayama Y, Seki $\mathrm{H}$, Itakura A, Takeda S. Placenta previa accreta spectrum; approach to reduce blood loss: intra-arterial balloon occlusion. In: Konishi I, Hiramatsu Y, Sakuragi N, Takeda S, eds. Surgery for Pregnancy with Placenta Previa and Placenta Accreta: Careful Preparation and Critical Management. OGS NOW, No.9 (in Japanese). Tokyo: Medical View; 2012:100-109. Copyright @ Medical View).

We have had four patients in whom we performed hemostatic sutures from the serosa side to the detached placenta surface with CIABO to avoid hysterectomy. We diagnosed the patients with placenta previa accreta clinically; however, we could not diagnose them pathologically using the placentas. If the patients were eagerly hoping to avoid hysterectomy, we could attempt it. However, since extensive placental invasion may cause massive bleeding, even in the case of strictly diagnosed placenta accreta, there should be no hesitation in performing hysterectomy at that time. Because intrauterine bleeding at the placenta detachment site is often from the vein, CIABO may be less effective than arterial bleeding (-Fig. $\mathbf{6}$ ).

11. Cut and ligate the round ligament and then the proper ligament of the ovary and the fallopian tube. Expand the retroperitoneal space.

The cut end of the uterine side and the distal side should be tightly ligated. It is said about all ligaments that bleeding from the cut end of the uterus side should not be ignored while performing hysterectomy during pregnancy because of increased circulatory blood volume. It is important that all cut ends should be ligated carefully to ensure that the knots do not slip. The peritoneum of the anterior lobe of the broad ligament should be incised up to a site near the bladder, and the posterior lobe of the broad ligament should be separated and incised in the direction toward the site of attachment of the uterosacral ligament to obtain a sheet of peritoneum. The location of the ureter should be definitely confirmed. ${ }^{2,3}$

12. Cut and ligate the uterine artery and vein and then cut and suture the uterosacral ligament and the cardinal ligament.
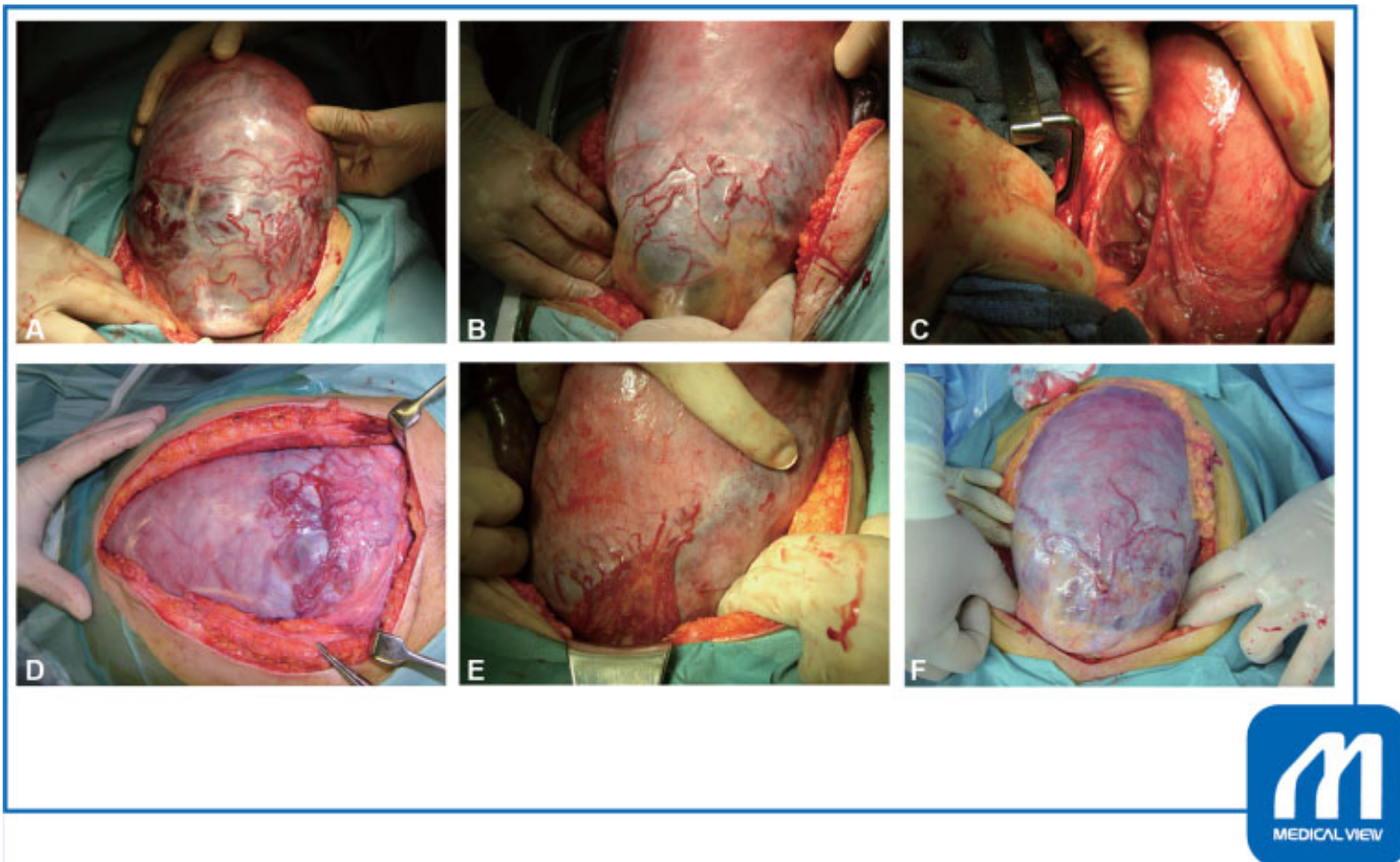

Fig. 6 Pictures of findings strongly suggesting invasion of the placenta. All the photos were from different patients who underwent hysterectomy and were diagnosed with placenta increta or percreta pathologically. (Reproduced with permission from Murayama Y, Seki $\mathrm{H}$, Itakura A, Takeda S. Placenta previa accreta spectrum; approach to reduce blood loss: intra-arterial balloon occlusion. In: Konishi I, Hiramatsu Y, Sakuragi N, Takeda S, eds. Surgery for Pregnancy with Placenta Previa and Placenta Accreta: Careful Preparation and Critical Management. OGS NOW, No.9 (in Japanese). Tokyo: Medical View; 2012:100-109. Copyright @ Medical View). 
The parametrium should be separated and cut with an electric cautery device, allowing the courses of the uterine artery and vein to be confirmed. The uterine artery and vein, including the upper part of the carinal ligament, should be cut and suture-ligated doubly with a 1-0 absorbable thread. Then, the uterosacral ligament and the remaining portion of cardinal ligament should be cut and suture-ligated, respectively. 2,3

13. Infuse 3,000 units of unfractionated heparin intravenously, immediately before balloon dilation.

We infused 3,000 units of unfractionated heparin intravenously immediately before balloon dilation to prevent thrombosis of the lower limbs. In our experience, using 6,000 units of unfractionated heparin before balloon dilatation may compromise blood coagulation, hence we now recommend 3,000 units of unfractionated heparin.

14. Dilate the balloon in both common iliac arteries.

The toughest procedures in $\mathrm{CH}$ are to separate the bladder and the uterus to cut the vaginal fornix. We inject the unfractionated heparin intravenously and dilate each side of the balloons in turn, just before starting to separate the bladder. With reference to the tourniquet guide in orthopedic surgery, we think that approximately 1 hour of occlusion can be tolerated; however, we deflate the balloons every 30 minutes just in case.

15. Confirm that the internal iliac artery pulsation becomes weak in the surgical field and that the pulse wave attenuates but does not disappear completely by oxygen saturation monitors attached to both the big toes.

The purpose of this procedure is to make sure that the balloons are correctly positioned in the common iliac artery. If the balloon enters the external iliac artery and is inflated there, it will increase the blood flow of the internal iliac artery and blood loss will also increase. In addition, it may cause unexpected lower limb ischemia. The point of using CIABO is that the blood flow to the intrapelvic region is significantly decreased, while that to the lower limbs is maintained at a low rate. Therefore, it is not necessary to dilate the balloons until the pulse wave completely disappears. The hemorrhage volume in the surgical field will decrease even when the wave attenuates. Conversely, if the pulse wave disappeared completely, it must be determined whether the balloons entered the external iliac artery.

16. Separate the urinary bladder from the uterus.

In cases of previous cesarean section complicated by placenta previa accreta, the placentas attach to the prior cesarean section scar, and there is abnormal vessel growth in the vesicouterine pouch. When these vessel growths are relatively mild, the completion of this procedure and hysterectomy in a short time will result in relatively low blood loss. In contrast, when the vessels swell extensively, this procedure should be performed carefully as follows. First, separate the sparse connective tissue between the uterine cervix and the bladder gently with the fingers; next, hold the clamp on the firm tissue between the fundus of the bladder and the uterine cervix; and then, cut and ligate it. Forced separation even if you feel a resistance may cause damage to the bladder.
Nevertheless, attention should be paid to the uterine muscular wall during this procedure to avoid damage. However, the procedure often causes damage to the bladder at the adhesion site. In such a case, the adhesion area and the wall of the bladder can be identified and there is no need to panic. We perform double-layer closure using absorbable surgical suture for the bladder injury as follows: the first layer is sutured one by one so that the mucosal and muscular layers are aligned; the second is a retention suture of the muscle that prevents the wound separation.

Regarding hysterectomy, it has also been reported that the posterior vaginal fornix is first opened from the Douglas fossa, the cardinal ligament and the uterine artery are ligated retrogradely, and finally, the uterus and bladder are separated. This method is effective to prevent damaging the bladder. However, in $\mathrm{CH}$, since the uterus of placenta previa accreta has a swollen bottom resembling the shape of a snowman, the approach from the Douglas fossa is rather difficult.

-Fig. 7 shows that the bladder separation is performed laterally in a patient with proliferative vessels of the peritoneum in the vesicouterine pouch. The incision of the anterior lobe of the broad ligament of the uterus is extended to the inguinal direction while avoiding the proliferated vessels in the vesicouterine pouch (- Fig. 7A).When separating the sparse connective tissue between the uterine cervix and the bladder, it is important to feel the presence of the uterine cervix and detach it gently with the fingers, while avoiding the elastic (the bladder) and firm (adhesion) tissues. - Fig. 7B shows that the separation finger going between the uterine cervix and the bladder meets in the middle. - Fig. 7C shows the further separation of the bladder from the uterus. We can see the bulging placenta through the uterus ( -Fig. 7C). - Fig. 7D shows the end of the separation of the bladder except for the peritoneum and the proliferative vessel of the vesicouterine pouch. The area of firm adhesion between the bladder and the penetrated placenta is dissected and held with a clamp (-Fig. 7E). When cutting the adhesive area, the cut end should be confirmed to be adequately apart from the fundus of the bladder. - Fig. 7F shows the ventral side of the removed uterus. The red and black bulge is the adhesion site of the placenta to the previous cesarean section scar. The pathological diagnosis is placenta percreta (-Fig. 7F).

17. Perform hysterectomy and close the amputation stump of the vagina.

The incision of the anterior vaginal fornix was performed using the palpation with the grasping forceps at the uterine cervix as a guide. We ligated the capillary plexus on the lateral side of the vagina and performed closed sutures of the amputation stump of the vagina. The surface of the bladder after separation from the uterus can have the over-swelling vessels. Therefore, care should be taken not to injure these vessels.

\section{Deflate the balloon.}

The balloons should be deflated on each side one after the other because this may cause hyperkalemia if the lower limb blood flow is completely blocked. After deflation of the 


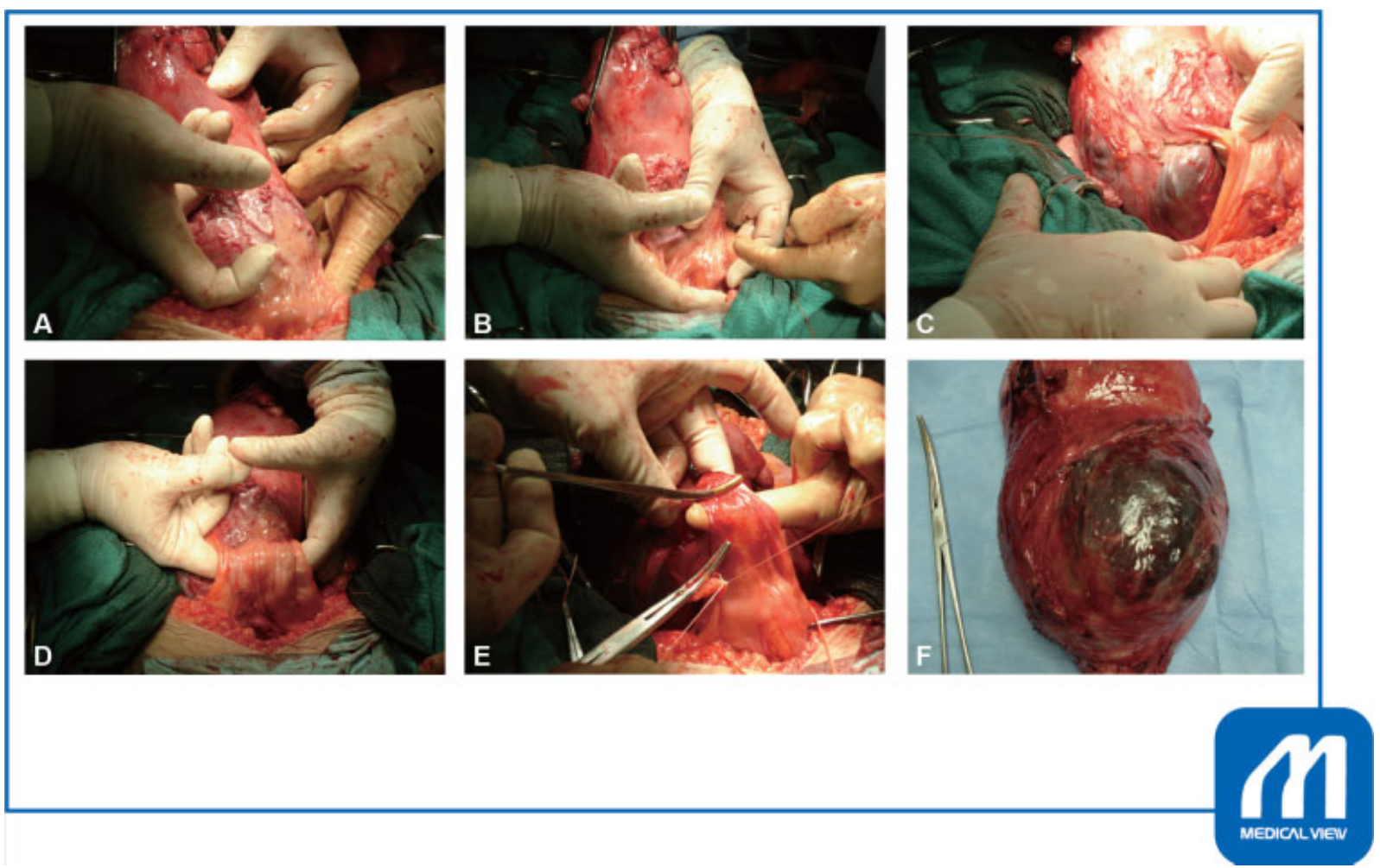

Fig. 7 (Reproduced with permission from Murayama Y, Seki H, Itakura A, Takeda S. Placenta previa accreta spectrum; approach to reduce blood loss: intra-arterial balloon occlusion. In: Konishi I, Hiramatsu Y, Sakuragi N, Takeda S, eds. Surgery for Pregnancy with Placenta Previa and Placenta Accreta: Careful Preparation and Critical Management. OGS NOW, No.9 (in Japanese). Tokyo: Medical View; 2012:100-109. Copyright (c) Medical View).

balloons, it is necessary to monitor the changes in T-waves on electrocardiogram and evaluate the potassium level of the arterial blood gas over time.

19. If necessary, use protamine sulfate for heparin neutralization.

If the activated coagulation time (ACT) value extends $>140$ seconds after deflating the balloons, you should consider using protamine sulfate for the neutralization of heparin.

20. Search for bleeding points and stop them. Close the incision of the abdomen.

After deflating the balloons, you should pay attention to bleeding from the cut end of the serosa or the cardinal ligament and from the bladder away from the uterus. If you find some bleeding points, perform hemostasis by ligation or electrocoagulation. After confirming that hemostasis has occurred, irrigate the surgical field with saline and then indwell the drain for the intrapelvic region before closing the abdominal incision.

\section{Remove the balloon and sheath introducers.}

We remove the balloon catheter soon after the end of $\mathrm{CH}$ (usually in the operating room) and remove the sheath introducer after ACT test to confirm no coagulation abnormality (usually in the patients' room). Hemostasis is performed by manually pressing the central side of the puncture site for $>10$ minutes. Subsequently, pressure hemostasis is continued using a sandbag for 5 hours. Since there is a case report of sudden hemorrhagic cardiac arrest due to internal hemorrhage from the femoral artery puncture site accumulating in the retroperitoneum, attention should be paid to changes in the blood pressure and heart rate by continuous monitoring and also to the condition of the puncture site, such as a hematoma and subcutaneous bleeding.

Conflict of Interest

None.

\section{References}

1 Murayama Y, Seki H, Itakura A, Takeda S. Placenta previa accreta spectrum; approach to reduce blood loss: intra-arterial balloon occlusion. In: Konishi I, Hiramatsu Y, Sakuragi N, Takeda S, eds. OGS NOW, No.9. Surgery for Pregnancy with Placenta Previa and Placenta Accreta: Careful Preparation and Critical Management (in Japanese). Tokyo: Medical View; 2012:100-109

2 Takeda S, Takeda J, Makino S. Cesarean section for placenta previa and placenta previa accreta spectrum. Surg J (N Y) 2020;06(S2) S110-S121

3 Takeda S, Takeda J, Murayama Y. Placenta previa accreta spectrum; cesarean hysterectomy. precision surgery in obstetrics and gynecology. Surg J (N Y) 2020. Doi: 10.1055/s-0040-1721492

4 Iwata A, Murayama Y, Itakura A, Baba K, Seki H, Takeda S. Limitations of internal iliac artery ligation for the reduction of intraoperative hemorrhage during cesarean hysterectomy in cases of placenta previa accreta. J Obstet Gynaecol Res 2010;36 (02):254-259

5 Shrivastava V, Nageotte M, Major C, Haydon M, Wing D. Casecontrol comparison of cesarean hysterectomy with and without prophylactic placement of intravascular balloon catheters for placenta accreta. Am J Obstet Gynecol 2007;197(04):402.e1-402.e5

6 Sumigama S, Itakura A, Ota T, et al. Placenta previa increta/percreta in Japan: a retrospective study of ultrasound findings, management and clinical course. J Obstet Gynaecol Res 2007;33(05):606-611 
7 Shih JC, Liu KL, Shyu MK. Temporary balloon occlusion of the common iliac artery: new approach to bleeding control during cesarean hysterectomy for placenta percreta. Am J Obstet Gynecol 2005;193(05):1756-1758

8 Ono Y, Murayama Y, Era S, et al. Study of the utility and problems of common iliac artery balloon occlusion for placenta previa with accreta. J Obstet Gynaecol Res 2018;44(03):456-462

9 Takeda J, Makino S. Temporary arterial balloon occlusion for obstetrical field. In: Takeda S, Kuwatsuru R, eds. Gynecologic and Obstetric Prophylactic Hemostasis by Intra-Arterial Balloon Occlusion. Singapore: Springer; 2018:33-39

10 He Q Li YL, Zhu MJ, et al. Prophylactic abdominal aortic balloon occlusion in patients with pernicious placenta previa during cesarean section: a systematic review and meta-analysis from randomized controlled trials. Arch Gynecol Obstet 2019;300(05):1131-1145

11 Shukunami K, Hattori K, Nishijima K, Kotsuji F. Transverse fundal uterine incision in a patient with placenta increta. J Matern Fetal Neonatal Med 2004;16(06):355-356 